\title{
Preface: Richard M. Wilson, Special issue honoring his 65th birthday
}

\author{
K. T. Arasu • Xiaoyu Liu - Gary McGuire
}

Published online: 1 September 2012

(C) Springer Science+Business Media, LLC 2012

This issue of the journal is dedicated to Professor Richard M. Wilson of California Institute of Technology on the occasion of his 65th birthday. The guest editors selected fifteen contributions covering various areas of Combinatorial Design Theory and Coding Theory; the contributors have close academic ties to Prof. R. M. Wilson. There does not seem to be better place than this to write a short biography on Prof. Wilson, highlighting his various milestones and achievements:

Richard M. (Rick) Wilson has been a key figure in combinatorics for over 40 years. He has made numerous contributions to the theory of combinatorial designs and finite geometries, to coding theory, and to extremal set theory.

Rick, as a second year graduate student, alongside his mentor D. K. Ray-Chaudhuri, found a solution of the (general) Kirkman School-girl problem in 1968. His 1969 thesis built on these methods, as well as recursive construction techniques developed by R. C. Bose, E. T. Parker, and S. S. Shrikhande, and H. Hanani. The thesis lead to a series of papers on the theory of combinatorial designs (in particular, pairwise balanced designs or PBDs). In addition to recursive constructions, the existence of difference families constructed from finite fields plays a crucial role. In 1974, he completed the proof of the "asymptotic" existence of 2-designs with fixed block size $k$. This work was recognized with the SIAM Polya Prize in 1975 (shared with R. P. Stanley and E. Szemeredi).

It would be difficult to find any papers, even today, on certain types of design theory which do not use ideas and notation introduced in these early, groundbreaking works.

\footnotetext{
K. T. Arasu $(\varangle) \cdot$ X. Liu

Department of Mathematics and Statistics, Wright State University, Dayton, OH 45435, USA e-mail:k.arasu@wright.edu

X. Liu

e-mail: xiaoyu.liu@wright.edu

G. McGuire

School of Mathematical Sciences, University College Dublin, Belfield Office Park-8, Belfield, Dublin 4, Ireland

e-mail: Gary.McGuire@ucd.ie
} 
Related to this work on 2-designs is Rick's work on sets of mutually orthogonal Latin squares (MOLS). His extensions of results by Bose, Parker, and Shrikhande have been further refined and lead to the best known lower bounds for the number of MOLS of order $n$.

Another early work was his proof with Ray-Chaudhuri of which is known as the Petrenjuk-Ray-Chaudhuri-Wilson inequality for $t$-designs. This was one of the inspirations for Ph. Delsarte's work on association schemes. This and other work on inequalities for $t$-designs, and on the existence of signed $t$-designs, relied on linear algebraic techniques and incidence matrices. The strong interest in linear algebra and incidence matrices has continued throughout Rick's career.

The Frankl-Wilson inequalities concerning families of sets with restricted pairwise intersection sizes appeared in 1982 and have significant consequences. In particular, it was used by J. Kahn and G. Kalai to disprove a conjecture of Borsuk. In 1984, Rick used matrix calculations in association algebras to give the first proof of the exact bound in the Erdös-Ko-Rado theorem.

Work on polynomial codes, on diagonal forms (or Smith forms) of incidence matrices related to uniform hypergraphs, and extremal problems has continued to today.

Deep results on asymptotic decompositions of edge-colored complete graphs, joint work with E. Lamken, A. Draganova, and Y. Mutoh, appeared in 2000 and 2008. Many seemingly diverse design-like problems can be put into this language and the corresponding existence questions immediately answered.

It is hardly necessary to mention the influence of the textbook titled "A Course in Combinatorics" written jointly with J. H. van Lint (with whom Rick collaborated in the 1980s in work on bounds on cyclic codes and Preparata-like codes). The book appeared in 1992, with a second edition in 2001, and became one of the standard graduate-level introductory texts in combinatorics. It is appreciated for its elegance and careful choice of topics, presenting significant results and "gems" from a wide variety of areas.

After 11 years on the faculty of The Ohio State University, Rick moved to Caltech in 1980 where he remains on the mathematics faculty. He has supervised numerous Ph.D. theses at both institutions, about 30 overall, and his students have followed a wide variety of career paths. He is known as an excellent teacher, at both the undergraduate and graduate level.

He has worked with a large number of distinguished coauthors, and has always freely shared ideas with his students and colleagues. A large number of papers may be found where he is credited with inspiration or supplying a proof.

Rick became interested in historical flutes in the late 1970s and has since avidly performed on, studied, and collected both original European flutes made in the 18th and 19th centuries, and replicas of flutes from this and earlier periods. He recently published, with A. Powell, a book on flute performance practice in London circa 1830. In the past decade, his interest has extended to traditional flute types of all countries, and he often accompanies poetry readings on shakuhachi and other world flutes.

Rick's wife Kathabela is a poet and an artist and they attend mathematical conferences together.

The guest editors believe that the papers appearing in this issue will stimulate further research in the topics covered. They are very grateful to all the contributing authors and the reviewers who helped make this issue possible. 